\title{
Objective Tinnitus Concomitant with Eye Blinking: A Case Report
}

\author{
Tae Hwan Kim, Ho Joon Jang, Soon Hyung Park, and Sung-II Nam \\ Department of Otolaryngology, School of Medicine, Keimyung University, Daegu, Korea
}

\section{Received March 4, 2015 \\ Revised April 19,2015 \\ Accepted June 17, 2015}

\author{
Address for correspondence \\ Sung-Il Nam, MD, PhD \\ Department of Otolaryngology, \\ School of Medicine, \\ Keimyung University, \\ 56 Dalseong-ro, Jung-gu, \\ Daegu 41931, Korea \\ Tel $+82-53-250-7715$ \\ Fax +82-53-256-0325 \\ E-mail entnamsi@dsmc.or.kr
}

Tinnitus is a common auditory phenomenon associated with many otological diseases, and is usually subjective. Objective tinnitus can be generated by para-auditory structures, usually derived from vascular or myogenic sources, or the eustachian tube. We present a rare case of intermittent unilateral tinnitus associated with eye blinking. Otoendoscopic examination showed that the external auditory canals and tympanic membranes were normal; however, rhythmic movements of both tympanic membranes, concomitant with the tinnitus, were evident whenever the patient blinked. The tympanometry and stapedial reflexes measured via impedance audiometry exhibited saw-tooth patterns; movement of the tympanic membrane was associated with eyelid blinking. The patient was managed conservatively, with reassurance and medication, and the condition became well-controlled. Here, we present this educational case and review the literature.

J Audiol Otol 2015;19(2):101-103

\section{Introduction}

Tinnitus is the perception of sound when an external stimulus is absent, and can be classified as either objective or subjective, depending on patient history and physical examination. Objective tinnitus can be triggered by vascular abnormalities, a patulous eustachian tube, abnormalities of the palatine or middle ear muscles, or defects in other structures. Tinnitus caused by middle-ear myoclonus is rare [1-6], and is usually assumed to develop secondarily to abnormal movement of the stapedius muscle or the tensor tympani. We present a rare case of myogenic tinnitus attributable to abnormal stapedius and/or tensor tympani muscle contraction, concomitant with eye blinking or eye closure. No facial palsy, hemifacial spasm, or blepharospasm was present.

\section{Case Report}

A 45-year-old female presented complaining of low-pitched

This is an Open Access article distributed under the terms of the Creative Commons Attribution Non-Commercial License (http://creativecommons. org/licenses/by-nc/3.0/) which permits unrestricted non-commercial use, distribution, and reproduction in any medium, provided the original work is properly cited. unilateral left-ear tinnitus 2 months in duration, which commenced during descent of an aircraft in which she was travelling. She had become increasingly worried when the tinnitus did not subside over time. The sound was present only when she blinked or closed her eyes, and occurred on every blink. The noise could not be easily characterized; it was a "combination of a buzz and chewing gum-like sound". No other ear symptom, such as otalgia, otorrhea, vertigo, or facial palsy, was evident. She had no history of any systemic illness, head trauma, or exposure of the ear to noise or any toxic drug. Her family history was unremarkable. She did not complain of facial pain or headache.

On otoendoscopic examination, the auricles, external auditory canals, and tympanic membranes of both ears were normal, but, whenever the patient blinked, to-and-fro movements of both tympanic membranes were evident, especially the posterior halves. Interestingly, she did not complain of rightside tinnitus; however, the right-side tympanic membrane also moved when she blinked (Fig. 1). Thus, we recorded left-side tinnitus that was not audible when a stethoscope was placed on the outer ear. Neither direct examination nor fiber-optic nasopharyngoscopy revealed any sign of palatal myoclonus (Supplementary Videos 1 and 2 in the online-only Data Sup- 
plement).

Pure tone audiometry showed that the hearing threshold was normal. Both tympanograms were of type A; the middleear pressure was normal when the patient did not blink (Fig.

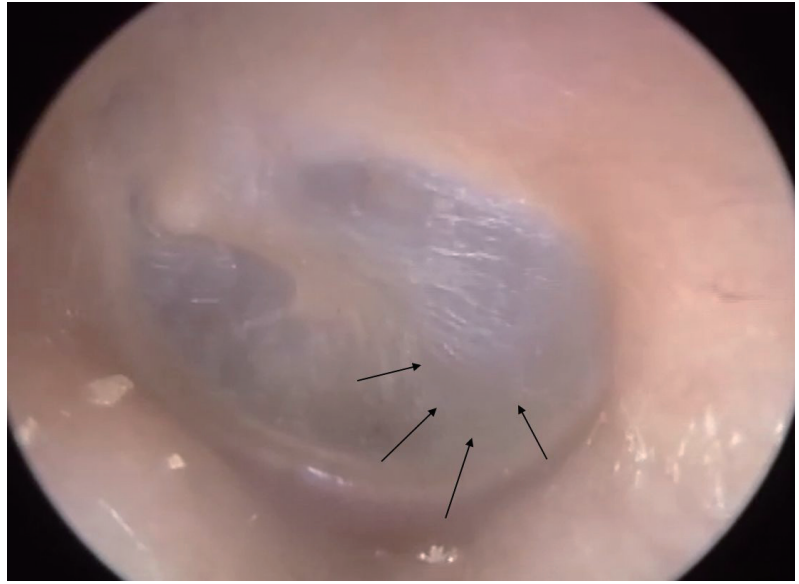

Fig. 1. The inward movement of the tympanic membrane was synchronous with eye blinking on otoscopic examination. The main moving portion of the left tympanic membrane was the postero-inferior quadrant (arrows).
2A). However, and interestingly, saw-tooth patterns associated with tympanic membrane movement were obtained during blinking (Fig. 2B). Acoustic reflex decay revealed irregular perturbations synchronous with eyelid blinking (Fig. 3). The tinnitus was considered to be attributable to stapedial muscle contraction, based on the reductive change in impedance audiometry compliance during blinking. The auditory brainstem response was normal. Temporal bone computed tomography (CT) revealed no abnormal lesion such as trauma, infection, or a vascular or neoplastic disease.

Initially, we explained the condition to the patient and reassured her over 2 weeks. However, the tinnitus persisted at the same intensity. After the 2 weeks, we prescribed carbamazepine $100 \mathrm{mg}$ orally twice daily. One week later, the tympanic membrane mobility was markedly reduced and the tinnitus well-controlled. On a visual analog scale, the tinnitus intensity fell from three to one.

\section{Discussion}

Among objective tinnitus, muscular tinnitus is rare and the

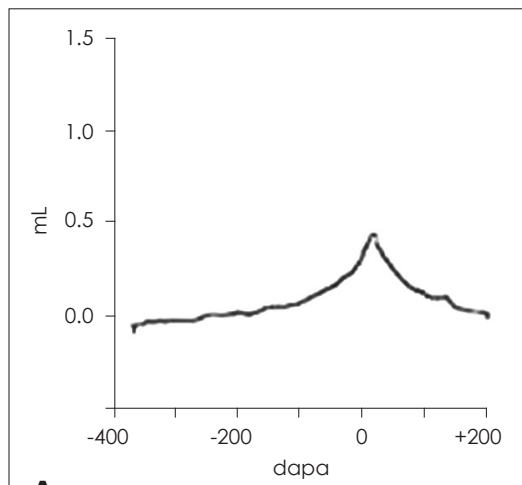

$A \leftarrow 600 / 200 \mathrm{daPa} / \mathrm{s}$

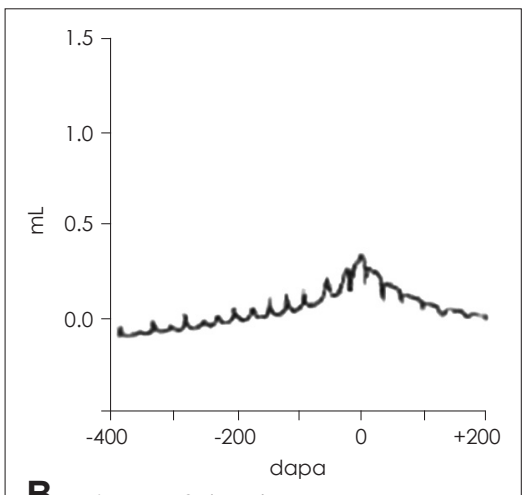

B $\leftarrow 50 \mathrm{daPa} / \mathrm{s}$
Fig. 2. A: Type A tympanogram shows normal middle ear pressure with normal mobility of the tympanic membrane without eye blinking. B: The saw-toothed pattern indicates movement of the tympanic membrane during eye blinking.

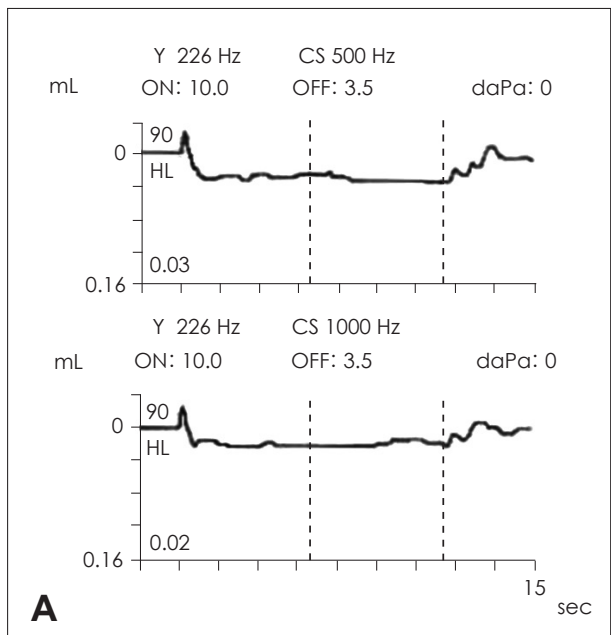

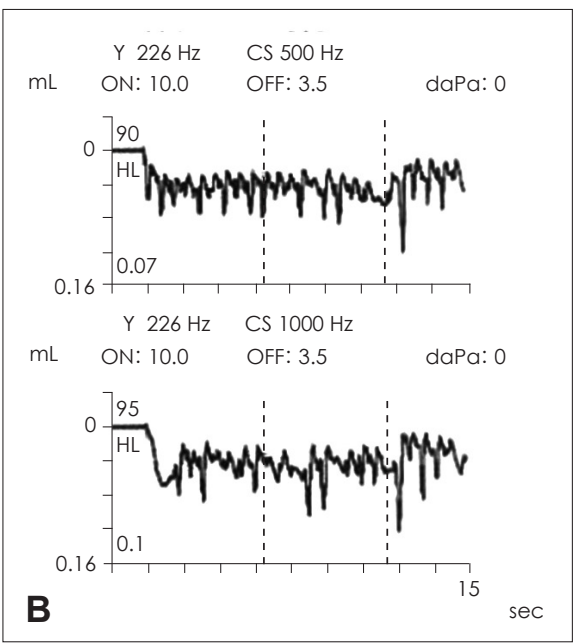

Fig. 3. Acoustic reflex decay showed (A) no perturbation without eye blinking and (B) sharp, irregular perturbation with eye blinking. 
precise mechanism poorly understood. Occasionally, a causative lesion can be identified in the brainstem or cerebellum via magnetic resonance imaging; however, most cases are idiopathic. Middle-ear muscular tinnitus is highly variable in nature, being most commonly described as crackling, but also as clicking, tapping, thumping, pulsatile, "(like a) fluttering moth", mechanical rumbling, buzzing, gushing, and whooping [1]. Stapedius muscle contraction is thought to produce a buzzing sound, and tensor tympani contraction a clicking [6]. Sound may also be caused by snapping or cracking of mucous membranes of the eustachian tube in response to peritubal muscle contraction [7], although the most frequent symptom is autophony [8]. Rock believed that the sounds were not intrusive in most subjects, being easily ignored; awareness of sound was closely associated with individual sensitivity [9]. In our patient, both tympanic membranes moved upon blinking, but she did not complain of right-side tinnitus.

Periodic movements of the tympanic membrane can often be seen upon otomicroscopy, and a rhythmic tremor of the soft palate may be noted upon flexible nasopharyngoscopic examination. The tremor may be recorded (for later evaluation) during conduct of acoustic reflex testing or impedance audiometry. No differential diagnosis of stapedius muscle contraction from tensor tympani contraction has yet been described, and, currently, no objective method exists by which the two conditions may be distinguished. Definitive diagnosis requires tympanotomy with direct exploration of contraction of the stapedius tendon or tensor tympani [10]. In our patient, the reductive change in impedance audiometry compliance during blinking was suggestive of stapedial muscle contraction.

Tinnitus associated with eyelid blinking is extremely rare, being first reported by Rajah [11]. The temporal and zygomatic branches of the facial nerve initiate eye closure. Facial nerve impulses directed to the orbicularis oculi muscle are presumed to spread abnormally toward the stapedial muscle. Such abnormal spread probably evokes stapedial muscle contraction. Several features of middle ear myogenic tinnitus suggest that the condition is of psychogenic origin [12]. However, the mechanism of abnormal spread remains unknown.

Several options exist for management of middle ear myoclonus. Initial treatment should feature reassurance and conservative therapy. East and Hazell [7] described tinnitusmasking devices and Chan and Palaniappan [13] developed zygomatic pressure maneuvers that should be used before resorting to medication or tenotomy.

Medical management with carbamazepine (up to $200 \mathrm{mg}$ three times daily) has been associated with variable degrees of success [14]. Close monitoring of potential side-effects such as bone marrow suppression, and development of liver and/or kidney abnormalities, is required. If conservative management is ineffective, botulinum toxin injections [15] or surgical resection of the middle ear muscle tendon are possible [1]. Thus, we reassured our patient and managed her medically.

We describe an unusual form of unilateral middle-ear muscular tinnitus concomitant with eyelid blinking, but in the absence of facial nerve palsy. Thorough history-taking, otoendoscopy, and stapedial reflex testing during eye closure, yielded important objective diagnostic data (particularly the irregular perturbations evident upon reflex testing); such workup is recommended when encountering this form of muscular tinnitus.

\section{Supplementary Materials}

The online-only Data Supplement is available with this article at http://dx.doi.org/10.7874/jao.2015.19.2.101.

\section{REFERENCES}

1) Badia L, Parikh A, Brookes GB. Management of middle ear myoclonus. J Laryngol Otol 1994;108:380-2.

2) Kim BG, Bang MY, Kim da H, Moon IS. Tinnitus related to eyelid blinking. Auris Nasus Larynx 2013;40:518-20.

3) Lee GH, Bae SC, Jin SG, Park KH, Yeo SW, Park SN. Middle ear myoclonus associated with forced eyelid closure in children: diagnosis and treatment outcome. Laryngoscope 2012;122:2071-5.

4) Marchiando A, Per-Lee JH, Jackson RT. Tinnitus due to idiopathic stapedial muscle spasm. Ear Nose Throat J 1983;62:8-13.

5) Park SN, Bae SC, Lee GH, Song JN, Park KH, Jeon EJ, et al. Clinical characteristics and therapeutic response of objective tinnitus due to middle ear myoclonus: a large case series. Laryngoscope 2013; 123:2516-20.

6) Watanabe I, Kumagami H, Tsuda Y. Tinnitus due to abnormal contraction of stapedial muscle. An abnormal phenomenon in the course of facial nerve paralysis and its audiological significance. ORL J Otorhinolaryngol Relat Spec 1974;36:217-26.

7) East CA, Hazell JW. The suppression of palatal (or intra-tympanic) myoclonus by tinnitus masking devices. A preliminary report. J Laryngol Otol 1987;101:1230-4.

8) O'Connor AF, Shea JJ. Autophony and the patulous eustachian tube. Laryngoscope 1981;91(9 Pt 1):1427-35.

9) Rock EH. Objective Tinnitus and the Tensor Tympani Muscle. Int Tinnitus J 1995;1:30-7.

10) Bhimrao SK, Masterson L, Baguley D. Systematic review of management strategies for middle ear myoclonus. Otolaryngol Head Neck Surg 2012;146:698-706.

11) Rajah V. Tinnitus related to eyelid blinking. J Laryngol Otol 1992; 106:44-5.

12) Williams DT, Ford B, Fahn S. Phenomenology and psychopathology related to psychogenic movement disorders. Adv Neurol 1995;65: 231-57.

13) Chan C, Palaniappan R. Middle ear myoclonus: a new technique for suppression of spontaneous clicking tinnitus. Int Tinnitus J 2010;16: 51-4.

14) Shea JJ, Harell M. Management of tinnitus aurium with lidocaine and carbamazepine. Laryngoscope 1978;88(9 Pt 1):1477-84.

15) Liu HB, Fan JP, Lin SZ, Zhao SW, Lin Z. Botox transient treatment of tinnitus due to stapedius myoclonus: case report. Clin Neurol Neurosurg 2011;113:57-8. 